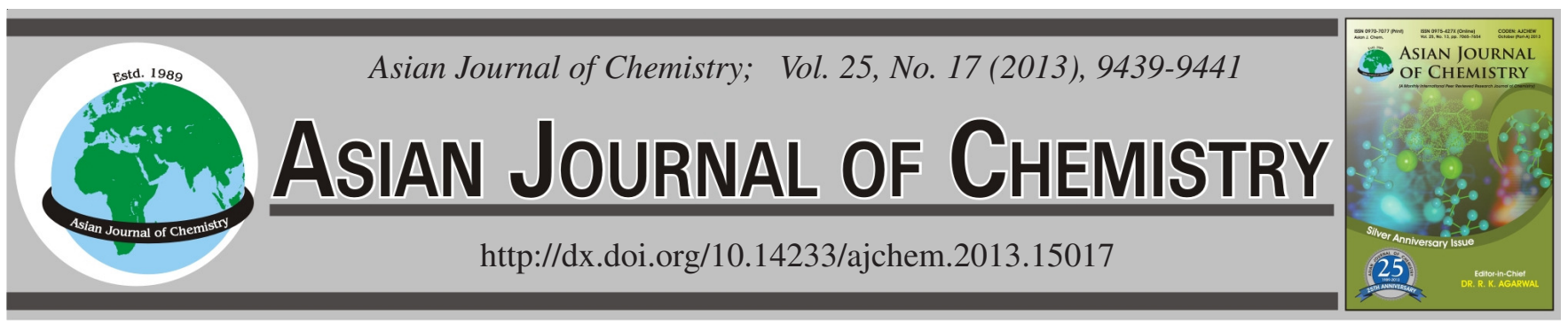

\title{
Determination of Calcium Metal in Calcium Cored Wire
}

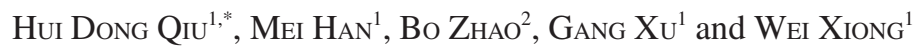
${ }^{1}$ Department of Chemistry and Chemical Technology, Chong Qing University of Science and Technology, Chongqing 401331, P.R. China
${ }^{2}$ Shang Hai Entry-Exit Inspection and Quarantine Bureau, P.R. China

*Corresponding author: Tel: +86 23 65023763; E-mail: qhd324@163.com

(Received: 24 December 2012;

Accepted: 1 October 2013)

AJC-14200

\begin{abstract}
The calcium analysis method in cored wire products is mainly measuring the total calcium content, including calcium oxide and calcium carbonate. According to the chemical composition of calcium cored wire and the chemical property of the active calcium in it, the gas volumetric method has been established for the determination of calcium content in calcium cored wire and a set of analysis device is also designed. Sampling in the air directly, intercepting the inner filling material of samples, reacting with water for 10 min, using standard comparison method and then the accurate calcium content can be available. The recovery rate of the experimental method was $93-100 \%$, the standard deviation is less than $0.4 \%$. The method is suitable for the determination the calcium in the calcium cored wire and containing active calcium fractions.
\end{abstract}

Key Words: Calcium cored wire, Active calcium, Gas volumetric method.

\section{INTRODUCTION}

The calcium cored wire ${ }^{1-4}$ is used to purify the molten steel, modify the form of inclusions, improve the casting and mechanical properties of the steel and also reduce the cost of steel. Because demanding technology conditions are required for the production of calcium cored wire products and there are the fake calcium cored wires with poor quality in the market, in which the calcium metal is not the real calcium metal, but the calcium compound such as calcium oxide, calcium carbonate etc, which is unnecessary. Thus it is significant to establish the method to determine the calcium metal in the calcium cored wire accurately.

The calcium cored wire generally contains $20 \%$ of the calcium metal, iron powder $75 \%$ and a small amount of impurities such as aluminum, silicon etc. The particle of the product shows nonuniform, soft and diffcult to break, moreover, the calcium metal can easily be oxidized and deliquescence because of the strong activity of the calcium, when the filling material was removed out. So it is difficult to take the usual method to prepare the sample. Zhou ${ }^{5}$ used the segment overall sampling analysis method and solved the problem. The filling material was treated with acid, then analyze the content of the calcium by complexometric titration with EDTA. This method determined the total calcium content rather than the available calcium content if the sample had the calcium compounds such as calcium oxide, calcium carbonate. Qi et al. ${ }^{6}$ used X-ray fluorescense spectrometry which can analyze the various state of the calcium. However, because the sample is heterogeneous and the amount of the sample for instrumental analysis is small, thus such sampling can not estimate characteristics of the whole ones accurately, so there are flaws to use the instrumental analysis to determine the calcium metal in calcium cored wire.

Since the chemical properties of each component in the calcium cored wire enormously vary, only the active calcium can rapidly react with water and generate a large mount of hydrogen, while the other components can't and the watersolubility of hydrogen is very low, so it is good to take the drainage and gas-collecting method to collect hydrogen and accurately measure the volume. Using standard comparison method to determine the active calcium content in calcium cored wire is reasonable. The methods used in the sample reaction device and measuring device are self-designed and it has also been applied for invention patent. The method for measuring calcium metal content is accurate, rapid and simple, reagents used mainly pure water is less, environmental friendly and the cost of analysis is low, moreover, device used in the instruments can be self-assembled, it is easy to popularize.

EXPERIMENTAL

Super thermostatic water bath (Jiangsu Guo Chuang Analytical Instrument Factory, Jiangyan, China) was stayed at a uniform temperature with eudiometer and reaction device. The reaction device and gas measurement device is selfassembly. Electronic balance $(0.1 \mathrm{mg}$, Sartorius China 
Company Limited, Beijing, China) is used for weighing the sample quality. The calcium $(99.99 \%)$ reacted with water to generate gas and took it as internal standard substance. Calcium cored wire samples are from the iron and steel enterprises (Shuicheng iron and steel Co., Ltd.).

Principle: The active calcium in calcium cored wire rapidly reacts with water at the room temperature and generates a large amount of hydrogen. And the drainage gas-collecting method is used to collect hydrogen and the volume is accurately determined. The standard comparison method is also used to detect the active calcium content in calcium cored wire.

Method: Fast and accurately weigh about internal powder sample from $5 \mathrm{~cm}$ long calcium cored wire, place it into a sample cup, fill the reaction device generator with water (saturated with hydrogen), screw plug, turn the piston of the eudiometer and connect the eudiometer with piston vent hole. Raise the leveling bottle (saturated with hydrogen), remove the air of the eudiometer. Put the reaction device into the super thermostatic water bath, turn the piston of eudiometer, connect the eudiometer with reaction device, maintain the consistent temperature of cooling water and tracheal interlayer water are same. The fixing ring of the sample cup is dipped in water. Gently shake the reaction device, which makes the sample and water react fully, once every two minutes. After $10 \mathrm{~min}$, the reaction is finished, check final reading again after $5 \mathrm{~min}$. If the two readings are same, which refer to the final volume reading of producing hydrogen. At the same time, take a certain amount of calcium particle with high purity (consistent with the gas volume of the samples produced), apply the same method to do experiment. Use standard comparison method to calculate the calcium metal content in calcium cored wire.

\section{RESULTS AND DISCUSSION}

Design of the experimental device: According to the experimental method, design the sample reaction device, measuring device and sealing liquid device. Reaction device is mainly made up of a special explosion-proof glass reaction vessel, a built-in rubber ring seal cover which is to sealing reaction container, while the sealing cover has the controllable injection rod, the bottom of which is rounded and can control the sample cup, put the reaction device into the super thermostat water bath in reaction, the resulting gas is up from the exhaust port of the sealing cover and then pass into the measurement device by the hose.

Measurement device is a double thermostatic eudiometer, the outer layer consists of super thermostat water bath in which the circulating water flows to keep the temperature and the wall of the inner one can read the gas volume (minimum scale interval is $2 \mathrm{~mL}$ ) and quality scale of the calcium. The top of the thermostatic eudiometer is controlled by two-way valve which is to adjust the sealing liquid level and to keep the pressure of inner layer and outer one same. The zero marking is in the upper of the eudiometer, the bottom is the sealing fluid outlet.

The sealing liquid device is the storage bottle which contains large amounts of the sealing liquid discharged by eudiometer. When the experiment is done, readjust level position of the sealing liquid and close the spring clip of over- flow. The sealing liquid is then pumped into the leveling bottle by the micropump and is injected into the eudiometer to adjust liquid level. The experimental device is given by Fig. 1.

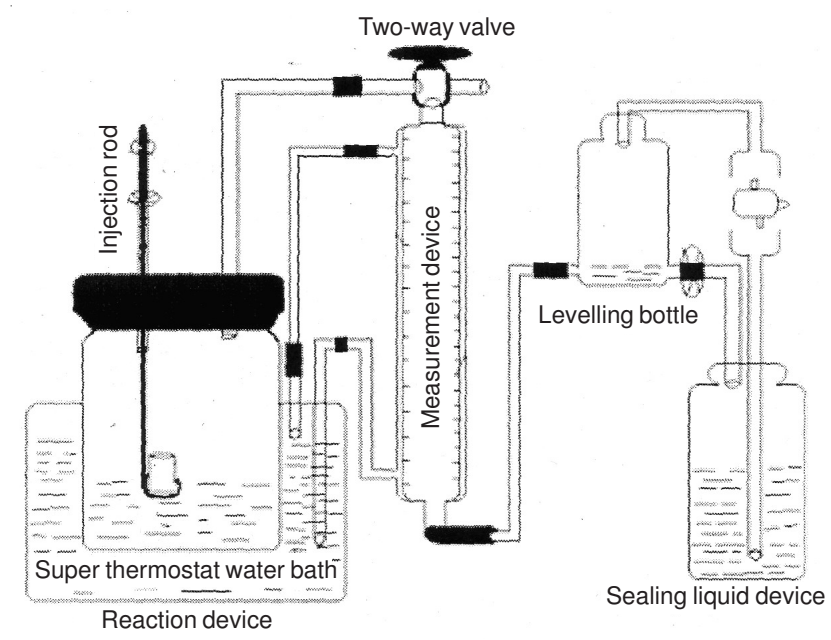

Fig. 1. Schematic diagram of experimental device

The reaction device designed in the experiment has a controllable injection rod which can control the rate of the reaction between sample and solution. And it has a good ability of sealing. The double-layer structure make the eudiometer maintain the temperature well, eliminate the influence on the volume of the gas from the environment. The sealing liquid can eliminate the reverse force of the gas volume with an overflow discharge liquid device of leveling bottle bottom and ensure the measurement accuracy of the gas volume.

Effects of sampling method and storage time: Because the chemical activity of the calcium is strong, the exposure to the air may produce oxidation and deliquescence. According to the reference ${ }^{7,8}$, the experiment adopts the sectional integral sampling analysis. Moreover, investigate the determination results of sampling methods between the glove box sampling in the protection of nitrogen and direct sampling in the air. It is showed that the experimental result deviation between the two methods is less than $0.2 \%$. Because the outside of the active calcium has a large number of iron powder which can protect the calcium metal from oxidizing by the air. Directly sampling and then keep them in the plastic bag within $2 \mathrm{~h}$. It can meet the basic requirements of chemical analysis.

Effects of reaction time: The reaction between the calcium having a high purity with water is very fast. However, in fact the reaction rate is slightly slow because the real samples of the calcium metal mix with iron powder. In order to ensure that the calcium metal in the real sample can react fully, the response of gas volume changes between 5-30 min have been discussed according to the experimental method.The experimental results are shown in Fig. 2.

The experiment results showed that calcium particle with high purity can react fully in $5 \mathrm{~min}$ and the calcium metal in calcium cored wire can react fully in $10 \mathrm{~min}$. So the reaction time determined is $10 \mathrm{~min}$. In order to prevent the hydrogen volume effecting from the exothermic reaction, read the gas volume and determine it as final volume after $5 \mathrm{~min}$. 


\begin{tabular}{ccccccccc}
\hline \multicolumn{8}{c}{ TABLE-1 } \\
\multicolumn{10}{c}{ ANALYTICAL RESULTS OF ACTIVITY CALCIUM IN IRON AND CALCIUM SAMPLES } \\
\hline Sample & \multicolumn{10}{c}{ Measure results } & \multicolumn{4}{c}{ Average (\%) } & SD \\
\hline 1\# & 15.9 & 16.1 & 15.9 & 16.3 & 16.4 & 15.8 & 16.1 & 0.24 \\
2\# & 18.1 & 18.3 & 17.4 & 17.9 & 18.2 & 18.4 & 18.1 & 0.36 \\
3\# & 18.8 & 18.9 & 18.2 & 19.1 & 19.3 & 18.7 & 18.8 & 0.38 \\
\hline
\end{tabular}

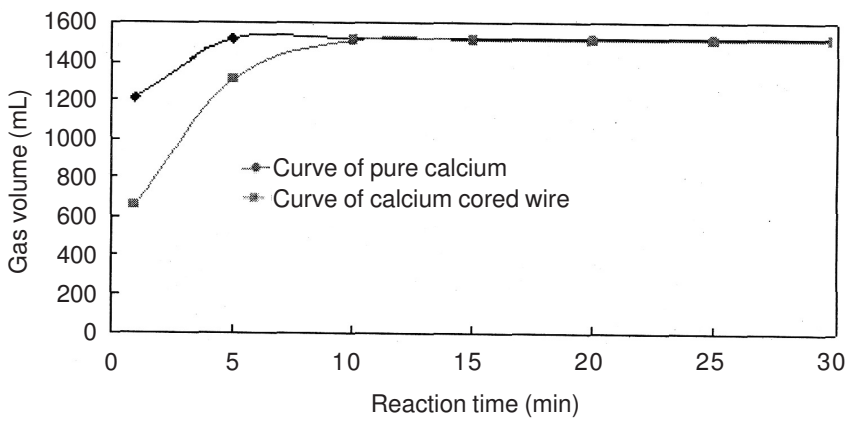

Fig. 2. Effect of reaction time

Effect of sealing liquid volume on gas volume: The gas produced by the reaction push the sealing liquid of the eudiometer into the leveling bottle, large sealing liquid discharged have a reverse force to the hydrogen gas volume in the eudiometer, which compress the hydrogen and make the reading small. In order to investigate reverse force effects from the sealing liquid and reduce the experiment error, different amounts of calcium are reacted with water, the results are shown in Fig. 3.

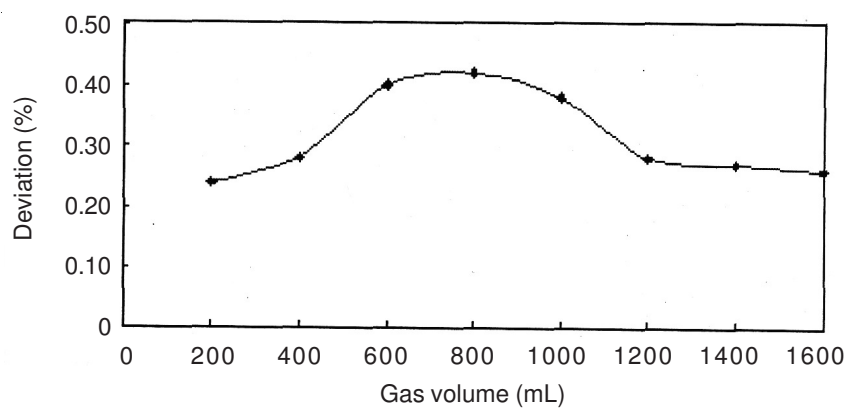

Fig. 3. Effect of sealing liquid volume to gas volume

Experiment results showed that, the volume of the sealing liquid discharged have a effect on hydrogen volume, maximum deviation is $0.42 \%$. In order to reduce measurement error, leveling bottle has a water outlet at the bottom. The sealing fluid discharged flows into the leveling bottle, then immediately goes out from the drainage port of the outflow of the leveling bottle. Such design would not produce reverse force on the gas generated, which eliminates the error from reverse gas compression of the sealing liquid.

Effect of environment temperature and atmospheric pressure: According to equation $\mathrm{PV}=\mathrm{nRT}$, gas volume will be affected by temperature and atmospheric pressure. In the non-standard condition, in order to ensure the accuracy of analysis results, experiment put the reaction device in the super thermostatic water bath and the temperatures of the eudiometer are controlled by the circulating water in the thermostatic bath. This can make the calcium with high purity and the calcium metal in the calcium cored wire react with water under the same conditions. So the experiment used the standard comparison method to eliminate the effects from the ambient temperature and atmospheric pressure on the gas volume measured in the reaction.

Effect of coexisting components: According to the YB/ T053-2000 standard, according to the theoretical analysis and experimental results, it is showed that the chemical component in the iron-calcium cored wire such as sulphur, phosphorus, carbon, ferrum, silicon and aluminium would not produce interference in the determination of calcium metal.

Sample analysis: According to the experimental method three samples were analyzed. The standard addition method is used to perform the recovery rate experiment. The experiment results are shown in Tables 1 and 2. The results shown that the content of calcium metal in calcium cored wire is less than $20 \%$ and which is basically agree with the products labeled, the standard deviation is less than $0.4 \%$, the recovery rate is from $93-100 \%$.

TABLE-2

RESULTS OF RECOVERY EXPERIMENT

\begin{tabular}{cccccc}
\hline $\begin{array}{c}\text { S. } \\
\text { No. }\end{array}$ & $\begin{array}{c}\text { Sample } \\
\text { mass }(\mathrm{g})\end{array}$ & $\begin{array}{c}\text { Measure } \\
\text { of } \mathrm{Ca}(\mathrm{g})\end{array}$ & $\begin{array}{c}\text { Add in } \\
\mathrm{Ca}(\mathrm{g})\end{array}$ & $\begin{array}{c}\text { Total of } \\
\mathrm{Ca}(\mathrm{g})\end{array}$ & $\begin{array}{c}\text { Recoveries } \\
(\mathrm{w} / \%)\end{array}$ \\
\hline 1 & 10.7501 & 1.7309 & 1.0208 & 2.7401 & 98.9 \\
2 & 10.1214 & 1.6295 & 1.1124 & 2.7290 & 98.8 \\
3 & 10.5106 & 1.6922 & 1.0791 & 2.6952 & 92.9 \\
4 & 9.7360 & 1.5675 & 1.0231 & 2.5214 & 93.2 \\
5 & 10.6182 & 1.7095 & 1.0187 & 2.7256 & 99.7 \\
6 & 9.2167 & 1.4839 & 1.0476 & 2.4936 & 96.4 \\
\hline
\end{tabular}

\section{Conclusion}

In this study, the experimental apparatus designed and assembled originally can measure the content of the calcium metal fast and accurately, which established the gas volumetric method for the determination of the calcium metal in the calcium cored wire. The recovery rate of the method is high while the standard deviation is small. So it is suitable for the analysis calcium metal of the calcium series products.

\section{REFERENCES}

1. F.M. Yuan, X.H. Wang and X.F. Yang, J. Univ. Sci. Technol. Beijing, Miner., Metall., Mater., 13, 486 (2006).

2. S. Basak, R. Kumar Dhal and G.G. Roy, Ironmaking Steelmaking, 37, 161 (2010).

3. J.X. Chen, Ferro-Alloy, 1, 1 (2007) (in Chinese)

4. Z.L. Yang and C. Song, An Hui Met., 2, 33 (2009) (in Chinese).

5. G.H. Zhou, Mei Shan Sci. Technol., 3, 41 (2008) (in Chinese).

6. Y. Qi, G.P. Ma and L. Ying, Metallurgical Anal., 25, 70 (2005) (in Chinese).

7. B. Zeng, J. Tu and Q. Fan, Special Steel Technol., 4, 49 (2011) (in Chinese).

8. J.H. Wang and F. Ma, Lai Gang Sci. Technol., 3, 27 (2010) (in Chinese). 